\title{
Die Erforschung der Sprechblase
}

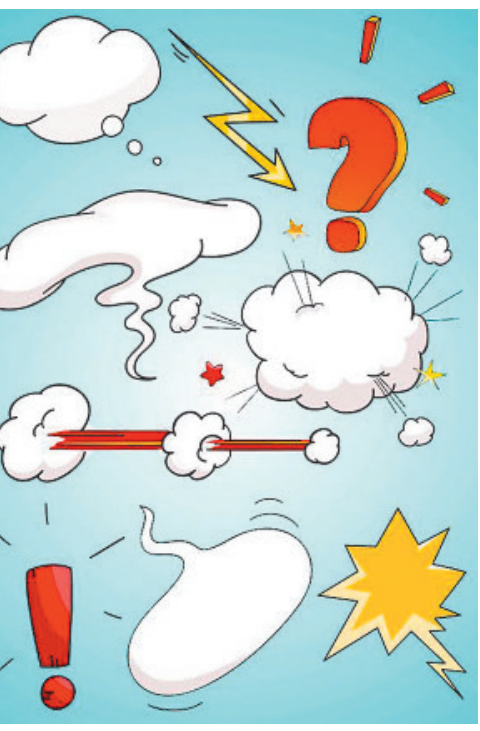

Alle Kollegen, denen ein ungenügender Impact Factor schlaflose Nächte bereitet, werden vor Neid erblassen. Kaum ein aktueller Artikel wurde in der Fach- und Laienpresse häufiger zitiert als der Beitrag einer Gruppe von Düsseldorfer Ärzten in «Acta Neurochirurgica». Es ging um 704 Fälle von Schädel-Hirntrauma als Folge tätlicher Gewalt, eingeordnet nach der Glasgow-Koma-Skala, veröffentlicht im Frühjahr 2011. Die Resultate wurden ausgiebig im deutschen und englischen Sprachraum kommentiert, im Radio erwähnt und im Fernsehen vorgestellt. «Zeit Online» titelte die Arbeit: «Asterix academicus - Forscher entdecken die Vorzüge eines bildgebenden Verfahrens». Initiant der Studie war der Hirnforscher und AsterixFan Marcel Kamp, der mit seinen Mitarbeitern aus 34 Bänden ein Risikoprofil von 690 tätlichen Angriffen auf römische Legionäre, Gallier, Piraten, Wikinger und zwei Schweizer erstellte. Gedacht war der Gag als Weihnachtsartikel für die jährliche Sonderausgabe des British Medical Journal, wo sie dann im Netz an Ostern veröffentlicht wurde. Diagnosekriterien waren eine seitlich heraushängende Zunge, der zeichnerisch dargestellte Grad der Bewusstseinseinschränkung, sowie Monokel- und Brillenhämatome. Tote oder schwere Schäden waren nicht nachweisbar. Dass Helme kluge Köpfe schützen, ist bekannt, vorausgesetzt, dass die Lederriemen optimal die Kopfbedeckung fixieren, was leider bei Cäsars Legionen nicht immer der Fall war. Die Erholung erfolgte erstaunlich schnell, obwohl die herrenlosen, meist auf dem Boden haftenden Sandalen, von der Wucht der erhaltenen Kinnhaken zeugen. Details, die natürlich jedem Leser bekannt sind, der auch die Begleitsymbole gezackter Blitze, läutender Glöckchen und schwarzer Wölkchen zu deuten weiss. Das mistelhaltige Dopingmittel aus dem Zauberkessel des Druiden Miraculix verleiht nicht nur ungeheure Kräfte, es fördert auch nachweisbar die Genesung, was die Pharmaindustrie interessieren dürfte.

Papa Moll und Globi sind medizinisch weniger ergiebig. Prellungen und eingebundene Daumen beschäftigen keine Intensivstation. Bei Tom und Jerry wird immer wild geprügelt bis die Katze flach an der Wand klebt, doch der Übergang vom 2-D- zum 3-DFormat bleibt immer noch wissenschaftliches Brachland. Verwundungen bei Spiderman und weiteren Superhelden kommen zahlreich vor, Batgirl endet im Rollstuhl, Wolverine verlor sein ganzes Skelett, Aquaman zweimal seine Hand, Thors Gesicht verunstalten wüste Narben, es gibt zerbrochene Wirbelsäulen, ausgerissene Arme und Beine und geblendete Augen. Die Medizinerzunft hat bisher die einfallsreiche Pro- thesentechnik der Comiczeichner nicht näher untersucht, dabei weiss man doch, wie genial Aussenseiter häufig sind.

Comicstrips sind wunderbare Projektionsflächen unserer Gesellschaft. Kein Wunder, dass die Welt der Sprechblasen ein häufiges Thema der Sekundärliteratur ist. Das Genre ist ungeheuer produktiv und kreativ, die Bildergeschichten haben sich seit Wilhelm Busch zu einer eigenen Kunstform entwickelt. Comics haben Sprache und Sehgewohnheiten mehr geprägt, als uns bewusst ist. Sie haben alle ideologischen und religiösen Gegner überwunden, Pädagogen und Moralapostel überrollt und sind dabei definitiv zur Weltsprache geworden. Die Werbung hat sie bereitwillig übernommen, sie sind eine gute Hilfe zur Erklä-

\section{«Asterix academicus - Forscher entdecken die Vorzüge eines bildgebenden Verfahrens»}

rung komplexer technischer und medizinischer Informationen für Laien und Fachpersonen. Für viele sind die Kunstfiguren realer als die Wirklichkeit. Viele Studien sind durchaus ernst gemeint und beackern mehr oder weniger originell das weite Feld der gezeichneten Sprache. Adam Scotts Figur «Dilbert» sagt mehr über unsere Bürowelt aus als manche dickleibigen Soziologenwerke, «Snoopy» und die Abenteuer von «Calvin and Hobbes» entführen uns auf unübertroffene Weise in die kindliche Fantasiewelt. Die Flut an Cartoons ist nicht mehr übersehbar, den wild wuchernden Dschungel einzuordnen, ist längst eine Aufgabe für Spezialisten. Werke der Weltliteratur von Goethe bis Hemingway, Dürrenmatt und Glauser werden in die Comicsprache übersetzt, Comics wie «Tintin» finden dieses Jahr den Weg ins Kino. Kameraeinstellungen haben die Zeichner angeregt und umgekehrt.

Die Geschichten von Asterix und Obelix wurden mit beigelegten Vokabulariumsblättern ins Lateinische übertragen, schwäbisch, bayerisch, fränkisch umgeschrieben und millionenfach in weit über einhundert Sprachen verkauft. Zum gleichen Universum gehören Fanclubs, Festivals, Lexika, Blogs und Sprachanalysen. Wer möchte sich da nicht auch einen Hinkelstein verdienen? Logisch, dass da Chirurgen auf der Erfolgswelle mitsurfen.

Erhard Taverna 